\title{
A sesame seeds-based breakfast could attenuate sub-clinical inflammation in type 2 diabetic patients: A randomized controlled trial
}

\author{
Zahra Bahadoran ${ }^{1}$, Parvin Mirmiran ${ }^{2}$, Somayeh Hosseinpour-Niazi ${ }^{1}$, Fereidoun Azizi ${ }^{3}$ \\ ${ }^{1}$ Nutrition and Endocrine Research Center, and Obesity Research Center, Research Institute for Endocrine Sciences, Shahid Beheshti \\ University of Medical Sciences, Tehran, Iran \\ ${ }^{2}$ Department of Clinical Nutrition and Dietetics, Faculty of Nutrition Sciences and Food Technology, National Nutrition and Food \\ Technology Research Institute, Shahid Beheshti University of Medical Sciences, Tehran, Iran \\ ${ }^{3}$ Endocrine Research Center, Research Institute for Endocrine Sciences, Shahid Beheshti University of Medical Sciences, Tehran, Iran
}

\section{Email address:}

mirmiran@endocrine.ac.ir (P. Mirmiran)

\section{To cite this article:}

Zahra Bahadoran, Parvin Mirmiran, Somayeh Hosseinpour-Niazi, Fereidoun Azizi. A Sesame Seeds-Based Breakfast Could Attenuate Sub-Clinical Inflammation in Type 2 Diabetic Patients: A Randomized Controlled Trial. International Journal of Nutrition and Food Sciences. Special Issue: Functional Foods and Nutraceuticals for Management of Type 2 Diabetes. Vol. 4, No. 2-1, 2015, pp. 1-5. doi: 10.11648/j.ijnfs.s.2015040201.11

\begin{abstract}
Background and aim: The aim of this study was to investigate the effects of Tahini, a traditional functional food made from ground un-hulled sesame seeds, on glucose homeostasis parameters and serum high-sensitive reactive protein (hsCRP) in diabetic patients. Methods: This randomized clinical trial included 41 patients with type 2 diabetes, who were randomly assigned to one of two groups: Group A (Tahini $30 \mathrm{~g} / \mathrm{d}, \mathrm{n}=21$ ) and group B (control, n=20). After an initial 2-week washout period, patients in group A, replaced a part of their usual breakfast with 2 tbsp ( 28 g) Tahini, while group B patients continued the usual breakfast meal for 6 weeks. Insulin and hs-CRP levels were measured, and insulin sensitivity index and homeostasis model assessment of insulin resistance index (HOMA-IR) at were calculated baseline and 6 weeks intervention. Results: After 6-weeks, there was a significant 21.1\% decrease in serum hs-CRP levels in group A. Slight but non-significant decrease in fasting serum glucose, serum insulin, insulin sensitivity index and HOMA-IR was observed during Tahini supplementation. Conclusion: Replacing a part of regular diet with Tahini as a functional food could attenuate sub-clinical inflammation in type 2 diabetic patients.
\end{abstract}

Keywords: High-Sensitive C Reactive Protein, Inflammation, Insulin Resistance, Sesame Seeds, Tahini, Type 2 Diabetes

\section{Introduction}

Systemic and sub-clinical inflammatory processes are considered as important pathogenic factor in the development of insulin resistance, type 2 diabetes and its complications especially vascular dysfunction and cardiovascular diseases $[1,2]$. Among proposed clinical markers of inflammation, high-sensitive C-reactive protein (hs-CRP), an acute phase 2 protein that releases from hepatocytes through stimulation by cytokines (interleukin-6, tumor-necrosis-factor-alpha and interleukin-1beta), was considered as a sensitive and reliable marker [3]. Even minor hs-CRP elevation has been shown to be associated with increased risk of cardiovascular events and clinically, serum hs-CRP levels $<1 \mathrm{mg} / \mathrm{L}, 1-3 \mathrm{mg} / \mathrm{L}$ and $>$
$3 \mathrm{mg} / \mathrm{L}$ have been considered as low, medium and high risk, respectively [4].

In patients with type 2 diabetes, hsCRP levels significantly correlate with hemoglobin $\mathrm{A} 1 \mathrm{C}$, lipid disorders, the stage of beta-cell dysfunction, insulin resistance and future cardiovascular risk; anti-inflammatory therapeutic approaches are now considered to be important in the management of type 2 diabetes and prevention of micro- and macro-vascular diseases $[5,6]$.

The major pharmacological approaches to attenuate inflammatory processes are oral anti-diabetic drugs and non diabetic drugs such as aspirin, statins, cyclooxygenase-2 
inhibitors, and fibrates; however use of some functional foods and their bioactive components may also be more effective. Sesame seed (seamen indicum L.) is one of these unique healthy foods with multiple beneficial effects including anti-aging, anti-cancer, anti-oxidative activity, antihypertensive, modulation of lipid metabolism and lipid peroxidation, enhancement of liver function, immunoregulatory and anti-thrombosis properties [7,8].

High level of mono and poly-unsaturated fatty acids, vitamin E, fiber, phytosterols, and other nutraceutical components such as bioactive lignans, sesamin, sesamol, episesamin and sesamolin have made sesame seeds and their byproducts functional foods beneficial in the prevention and treatment of chronic disease and metabolic disorder $[9,10]$. Anti-inflammatory properties of sesame seed and its byproducts have been shown in animal models [11,12], however in humans these effects have seldom been studied $[13,14]$.

In this study we assessed the effects of 6-week consumption (in breakfast meal) of Tahini, a traditional food stuff produced from grounded un-hulled sesame seeds (seamen indicum L.), on fasting serum glucose, serum insulin, homeostasis model assessment of insulin resistance index (HOMA-IR), and serum hs-CRP as inflammatory marker in type 2 diabetic patients.

\section{Material and Methods}

\subsection{Patients and Study Design}

This was a parallel, randomized, and controlled study. Type 2 diabetic patients, aged 30-60 years with a clinical diagnosis of type 2 diabetes (for at least one year) were recruited from the Iran Diabetes Society; they were excluded if they had severe impairment of cardiac, hepatic or renal function, gestation or lactation or if they used insulin injection or consumed antioxidant supplements. Forty-one patients, initially eligible, were included in the study. Subjects were randomly assigned to one of two groups: Group A (Tahini $28 \mathrm{~g} / \mathrm{d}, \mathrm{n}=21$ ) and group B (control, $\mathrm{n}=20$ ). Random allocation of patients to treatment groups was performed by sequentially numbered containers. Randomization was performed by an assistant and group allocation was blinded for the investigator and participants.

Ethics approval for the trial was obtained from ethical committee of the Research Institute for Endocrine Sciences of the Shahid Beheshti University of Medical Sciences. This clinical trial registered in the "Iranian Registry of Clinical Trials" at http://www.irct.ir with the following identification: IRCT201105161640N4, was conducted between March and July 2011. The methods and results of the current study are reported according to Consolidated Standards of Reporting Trials guidelines [15].

\subsection{Interventions}

During an initial 2-week washout period following recruitment, energy content of breakfast meal was adjusted for all patients within the range of $270 \mathrm{kcal}$, without any changes in regular breakfast pattern. The patients were advised to avoid intake of foods containing sesame seeds or sesame oils. After this period, patients in group A, replaced a part of their usual breakfast with 2 tbsp ( $\sim 28 \mathrm{~g})$ Tahini, so that energy content of breakfast be maintained in the same range; in group B patients continued with the usual breakfast meal. Each patient in group A received 2 boxes (1200 g) of Tahini (grounded un-hulled sesame seeds without any additives) purchased from Oghab Halva Company (Tehran, Iran). Patients were recommended to maintain their regular lifestyle, including diet and physical activity throughout the study period. Patients were contacted every week to evaluate compliance to the intervention and to enquire regarding any possible side effects such as allergic reactions.

\subsection{Measurements}

To monitor dietary compliance and assess nutrient intakes three-day dietary recalls, including 2 weekdays and 1 weekend day, were collected at baseline and again after 6 weeks from subjects,. Weight, height, and waist circumference were measured at baseline and 6 weeks later. Weight was measured to the nearest $100 \mathrm{~g}$ with digital scales, while the subjects were minimally clothed without shoes. Height was measured to the nearest $0.5 \mathrm{~cm}$, in a standing position without shoes, using a tape meter. Waist circumference was measured to the nearest $0.1 \mathrm{~cm}$, at the umbilical level and that of the hip, at the maximum level, over light clothing, using an unstretched tape meter, without any pressure to the body. Body mass index was calculated as weight $(\mathrm{kg})$ divided by square of the height $(\mathrm{m} 2)$. For blood pressure measurements, after a 15-minute rest in the sitting position, two measurements of blood pressure were taken, on the right arm, using a standardized mercury sphygmomanometer; the mean of the two measurements was considered as the participant's blood pressure.

\subsection{Biochemical Analysis}

At baseline and again 6 weeks after intervention, 12-h fasting blood samples were collected into tubes containing $0.1 \%$ EDTA and centrifuged at $4^{\circ} \mathrm{C}$ and $500 \mathrm{~g}$ for $10 \mathrm{~min}$ to separate serum. Serum frozen at $-70^{\circ} \mathrm{C}$, on the day of blood collection for biochemical analysis. Fasting plasma glucose was measured by the enzymatic colorimetric method using a glucose oxidation kit (Pars Azmun Company, Tehran, Iran). Serum insulin concentration was measured by using enzymelinked immunosorbent assay (ELISAs) kit (Mercodia, Uppsala, Sweden). The fasting glucose to insulin ratio was estimated as the insulin sensitivity index [16]. Insulin resistance was estimated using the homeostasis model assessment of insulin resistance (HOMA-IR) index, which is defined as fasting plasma insulin ( $\mathrm{mU} / \mathrm{l})$ times fasting plasma glucose (mmol/l) divided by 22.5 [17]. Serum hs-CRP (pg/ml) concentration was measured by using enzyme- linked immunosorbent assay (ELISA) kit (Diagnostics Biochem Canada Inc., Ontario, Canada). The intra assay coefficient of 
variations of all assays was $<5 \%$.

\subsection{Statistical Methods}

The sample size was calculated to detect a difference among groups in serum hs-CRP with $95 \% \mathrm{CI}$ and $90 \%$ power; sample size with regard to the possible loss of the samples was calculated as 20 patients in each group. Statistical analysis was performed with SPSS (version 16.0; SPSS, Inc., Chicago, IL, USA). The Kolmogorov-Smirnov test was used to test for a normal distribution. Differences between the 2 groups at baseline were tested with student's t-test. Paired $t$ test was used to compare baseline and 6-week values in each group. P values $<0.05$ were considered significant.

\section{Results}

Of forty-one randomized patients, 36 completed the study [group A, $\mathrm{n}=20$ and group $\mathrm{B}, \mathrm{n}=16$ ] and were included in the analysis. Baseline characteristics of study participants are shown in Table 1. The mean age of participants was $50 \pm 10$ and $52 \pm 9 \mathrm{y}$, and duration of diabetes was 7.6 \pm 5.5 and $7.4 \pm 4.4$ $\mathrm{y}$, in groups $\mathrm{A}$ and $\mathrm{B}$ respectively. There were no significant differences between the groups for age, duration of diabetes and other anthropometrics and clinical measurements at baseline.

Table 1. Baseline characteristics of participants

\begin{tabular}{lcc}
\hline & \multicolumn{2}{c}{ Baseline values } \\
& Tahini $(\boldsymbol{n = 2 0})$ & Control $(\boldsymbol{n}=\mathbf{1 6})$ \\
\hline Age, $y$ & $50 \pm 10$ & $52 \pm 9$ \\
Sex, $M / F$ & $4 / 16$ & $4 / 12$ \\
Duration of diabetes, $y$ & $7.6 \pm 5.5$ & $7.4 \pm 4.4$ \\
Weight, $\mathrm{kg}$ & $72.3 \pm 9.6$ & $74.8 \pm 9.7$ \\
Body mass index, $\mathrm{kg} / \mathrm{m}^{2}$ & $28.5 \pm 3.1$ & $29.9 \pm 3.4$ \\
Waist circumference, $\mathrm{cm}$ & $93 \pm 6.9$ & $98 \pm 8.2$ \\
Systolic blood pressure, $\mathrm{mmHg}$ & $116 \pm 18$ & $125 \pm 15$ \\
Diastolic blood pressure, $\mathrm{mmHg}$ & $78 \pm 10$ & $81 \pm 8$ \\
\hline
\end{tabular}

${ }^{1}$ Data are mean \pm SD (No significant differences were observed between the groups

using the independent sample t-test.

A regular breakfast meal for most patients, before the study, was bread and cheese; this pattern changed to bread and Tahini for the intervention group, and remained unchanged for controls during the study. Breakfast patterns in both groups had similar energy content of approximately $270 \mathrm{kcal}$. Nutritional composition of Tahini is presented in Table 2. Energy content of $28 \mathrm{~g}$ Tahini was approximately $172 \mathrm{kcal}$; carbohydrate, protein and total fat also were $5.1 \mathrm{~g}, 5.07 \mathrm{~g}$, and $16 \mathrm{~g}$, respectively. The nutrients content of both breakfast patterns (calculated using the Nutritionist Software version 4) is shown in Table 3. As compared to control pattern, Tahini consumption increased fat (total fat, mono-unsaturated fat, and poly-unsaturated fat) and fiber, and decreased saturated fat content of breakfast meal in group A. Based on the patient reports, lifestyle and medication status remained unchanged during the study period. Subject compliance was over $88 \%$ for $92 \%$ for Tahini (based on remaining weight of Tahini at the end of treatment period) and control groups, respectively. None of the patients in group A reported any adverse effects from consumption of Tahini.

Table 2. Nutritional composition of Tahini (paste of ground un-hulled sesame seeds)

\begin{tabular}{ll}
\hline Nutritional composition & Tahini $(\mathbf{2 8} \mathbf{g})$ \\
\hline Energy, $k$ cal & 172 \\
Carbohydrate, $g$ & 5.1 \\
Protein, $g$ & 5.07 \\
Total fat, $g$ & 16 \\
Saturated fat, $g$ & 2.24 \\
Mono-unsaturated fat, $g$ & 6.04 \\
Poly-unsaturated fat, $g$ & 7.01 \\
Dietary fiber, $g$ & 2.6 \\
\hline
\end{tabular}

Table 3. Comparison of the nutritional composition of the breakfast meal patterns in intervention and control group

\begin{tabular}{lcc}
\hline & \multicolumn{2}{c}{ Baseline values } \\
& Intervention $(\boldsymbol{n}=\mathbf{2 0})$ & Control $(\boldsymbol{n}=\mathbf{1 6})$ \\
\hline Wheat bread, $g$ & 30 & 60 \\
Cheese, $g$ & - & 30 \\
Ardeh ${ }^{*}, g$ & 28 & - \\
Tea, cup & 1 & 1 \\
Sugar, $g$ & 5 & 5 \\
Energy, kcal & 271 & 269 \\
Carbohydrate, $g$ & 29.06 & 42.5 \\
Protein, $g$ & 7.54 & 9.8 \\
Total fat, $g$ & 15.24 & 6.7 \\
Saturated fat, $g$ & 2.24 & 4.47 \\
Mono-unsaturated fat, $g$ & 6.04 & 1.38 \\
Poly-unsaturated fat, $g$ & 7.01 & 0.18 \\
Dietary fiber, $g$ & 3.21 & 1.3 \\
\hline
\end{tabular}

* Ardeh; paste of ground un-hulled sesame seeds

Nutritional composition of both breakfast meal was determined using the

Nutritionist software, version 4.

Comparison between serum levels of fasting glucose, insulin, hs-CRP and glucose homeostasis parameters, before and after intervention in both groups group are shown in Table 4. After 6 weeks, there was a significant $21.1 \%$ decrease in serum hs-CRP levels in group A. More slightly but non-significant decrease in fasting serum glucose, serum insulin, insulin sensitivity index and HOMA-IR also were observed during Tahini supplementation. Anthropometric measures and blood pressure were unchanged throughout the study period in both groups (data are not shown). 
Table 4. Serum levels of fasting glucose, insulin, hs-CRP and glucose homeostasis parameters in intervention and control group ${ }^{1}$

\begin{tabular}{lcccccccc}
\hline & \multicolumn{3}{c}{ Intervention $(\boldsymbol{n}=\mathbf{2 0})$} & & \multicolumn{3}{c}{ Control $(\boldsymbol{n}=\mathbf{1 6})$} \\
& \multicolumn{2}{c}{ Baseline } & \multicolumn{2}{c}{ 6 Weeks } & & Baseline & 6 Weeks \\
\hline Fasting glucose, $m$ mmol/l & Mean & SD & Mean & SD & Mean & SD & Mean & SD \\
Fasting serum insulin, $m U / l$ & 8.56 & 2.83 & 7.68 & 2.87 & 8.39 & 2.89 & 8.72 & 2.82 \\
Insulin sensitivity index & 10.22 & 6.89 & 9.69 & 4.50 & 8.85 & 2.73 & 9.22 & 7.55 \\
HOMA-IR & 1.24 & 0.91 & 1.01 & 0.60 & 1.01 & 0.41 & 1.31 & 0.74 \\
hs-CRP, $\mu g / m l$ & 3.82 & 3.60 & 3.13 & 1.43 & 3.39 & 1.75 & 3.43 & 2.34 \\
& 4.76 & 2.35 & $3.48^{2}$ & 2.21 & 3.01 & 2.73 & 3.04 & 2.42 \\
\hline
\end{tabular}

${ }^{1}$ Data are mean $\pm \mathrm{SD}$

${ }^{2}$ Significantly different from baseline (paired t test; $P<0.05$ )

HOMA-IR, homeostasis model assessment of insulin resistance; hs-CRP, High-sensitive C reactive protein.

\section{Discussion}

The results from this randomized clinical trial showed that replacement of $28 \mathrm{~g} / \mathrm{d}$ Tahini in breakfast meal, for 6 weeks, led to significant decrease in serum levels of hs-CRP, as a sensitive marker of sub-clinical systemic inflammation, in type 2 diabetic patients. Since the attenuation of inflammatory processes in diabetic patients is considered as a key therapeutic approach to prevent progression of undesirable complications, so this favorable capacity of sesame seeds may be clinically valuable.

There are limited and inconsistent studies investigated the anti-inflammatory capacity of sesame seed and it's by products; five weeks supplementation of overweight and obese men with $25 \mathrm{~g} / \mathrm{d}$ sesame ( $\sim 50 \mathrm{mg} / \mathrm{d}$ sesame lignan) had not beneficial effects on systemic inflammatory markers including C-reactive protein, interleukin-6, tumor necrosis factor- $\alpha$ [13]. Recently, Kratzi et al reported that consumption of $35 \mathrm{~g} / \mathrm{d}$ sesame oil had not favorable postprandial and long-term effects on serum CRP levels [14]; however previously anti-inflammatory potential of sesame had been reported in animal models $[11,12]$. It seems that anti-inflammatory properties of sesame seeds and its by products are mainly associated with bioactive components including lignans; Sesamin and sesamolin, main sesame lignans, could reduce activity of p38 mitogen-activated protein kinase (MAPK) and nuclear factor (NF)-kappaB [18]; enhanced activity of p38 MAPK and NF- $\mathrm{B}$ have key roles in the development of inflammation in diabetes, and inhibitors of these pathways could interact with production of inflammatory mediators including IL- 1 , TNF- $\alpha$ and IL- 6 and prevent diabetes development and its long-term complications [19,20]. Another mechanism could explain anti-inflammatory properties of sesame seed is that sesame lignans act as natural ligands for peroxisome proliferatoractivated receptor gamma (PPAR- $\gamma)[21]$; treatment with PPAR- $\gamma$ agonist has lead to substantial reduction of hs-CRP and other cardiovascular risk markers [22].

In this study we did not observed significant improvement in glucose homeostasis parameters after supplementation with Tahini in type 2 diabetic patients; however beneficial effects of sesame seeds and sesame oil on glycemic control have been previously reported by some studies. In one controlled and open-label clinical trial, treatment of type 2 diabetic women with defatted sesame flour was accompanied with better glycemic control [23]; administration of $35 \mathrm{~g} / \mathrm{d}$ sesame oil for 45 days led to significant decrease of blood glucose and hemoglobin A1c in diabetic patients [24]. In streptozotocin-induced diabetic rats, supplementation of a commercial diet with $6 \%$ sesame oil for 42 days significantly reduced blood glucose and serum levels of glycated hemoglobin [25].

To our knowledge this was a first study indicated the effects of Tahini on insulin resistance and inflammation in type 2 diabetic patients; however like other studies this clinical trial had few limitations including sample size was small, study duration was short and only one sample was obtained following the intervention. Further studies with longer duration and various doses may shed more light on the importance of the anti-inflammatory therapeutic effects of Tahini in diabetic patients. In the present study, the effects of Tahini, as a traditional and unique functional food, on glucose homeostasis parameters and serum hs-CRP in type 2 diabetic patients were investigated; the results showed that replacing a part of regular diet with Tahini could have favorable effects on modulation of inflammation in these patients.

\section{Acknowledgments}

This study was funded by the Research Institute of Endocrine Sciences, Shahid Beheshti University of Medical Sciences, Tehran, Iran. The authors express appreciation to the participants of this study and would like to acknowledge Ms Niloofar Shiva for language editing of the manuscript. None of the authors had any personal or financial conflicts of interest.

\section{References}

[1] Ndumele CE, Pradhan AD, Ridker PM. (2006). Interrelationships between inflammation, C-reactive protein, and insulin resistance. Journal of the Cardiometabolic Syndrome 1:190-6.

[2] Ridker PM. (2004). High-sensitivity C-reactive protein, inflammation, and cardiovascular risk: from concept to clinical practice to clinical benefit. American Heart Journal 148(1 Suppl):S19-26. 
[3] Nyström T. (2007). C-reactive protein: a marker or a player? Clinical Science (Lond) 113:79-81.

[4] Castoldi G, Galimberti S, Riva C, Papagna R, Querci F, Casati M, Zerbini G, Caccianiga G, Ferrarese C, Baldoni M, Valsecchi MG, Stella A. (2007). Association between serum values of C-reactive protein and cytokine production in whole blood of patients with type 2 diabetes. Clinical Science (Lond) 113:103-8.

[5] Sarangi R, Padhi S, Mohapatra S, Swain S, Padhy RK, Mandal MK, Patro SK, Kumar S. (2012). Serum high sensitivity C-reactive protein, nitric oxide metabolites, plasma fibrinogen, and lipid parameters in Indian type 2 diabetic males. Diabetes and Metabolic Syndrome 6:9-14.

[6] Roopakala MS, Pawan HR, Krishnamurthy U, Wilma Delphine Silvia CR, Eshwarappa M, Prasanna Kumar KM. (2012). Evaluation of high sensitivity C-reactive protein and glycated hemoglobin levels in diabetic nephropathy. Saudi Journal of Kidney Diseases and Transplantation 23:286-9.

[7] Martinchik AN. (2011). Nutritional value of sesame seeds. Vopr Pitan 80:41-3.

[8] Namiki M.(2007). Nutraceutical functions of sesame: a review. Critical Reviews in Food Science and Nutrition 47:651-73.

[9] Elleuch M, Besbes S, Roiseux O, Blecker C, Attia H. (2007). Quality characteristics of sesame seeds and by-products. Food Chemistry 103:641-50.

[10] Kamal-Eldin A, Moazzami A, Washi S. (2011). Sesame seed lignans: potent physiological modulators and possible ingredients in functional foods \& nutraceuticals. Recent Patents on Food, Nutrition \& Agriculture 3:17-29.

[11] Hsu DZ, Chen KT, Chu PY, Li YH, Liu MY. (2007). Sesame oil protects against leadplus- lipopolysaccharide-induced acute hepatic injury. Shock 27:334-337.

[12] Hsu DZ, Su SB, Chien SP, Chiang PJ, Li YH, Lo YJ, Liu MY. (2005). Effect of sesame oil on oxidativestress- associated renal injury in endotoxemic rats: involvement of nitric oxide and proinflammatory cytokines. Shock 24:276-280.

[13] Wu JH, Hodgson JM, Puddey IB, Belski R, Burke V, Croft KD. (2009). Sesame supplementation does not improve cardiovascular disease risk markers in overweight men and women. Nutrition, Metabolism and Cardiovascular Disease 19:774-8.

[14] Karatzi K, Stamatelopoulos K, Lykka M, Mantzouratou P, Skalidi S, Manios E, Georgiopoulos G, Zakopoulos N, Papamichael C, Sidossis LS. (2012). Acute and long-term hemodynamic effects of sesame oil consumption in hypertensive men. Journal of Clinical Hypertension (Greenwich) 14:630-6.

[15] Schulz KF, Altman DG, Moher. (2010). CONSORT 2010 statement: updated guidelines for reporting parallel group randomized trials. Journal of Clinical Epidemiology 63:83440 .

[16] Silfen ME, Mavibo AM, McMahon, Levine LS, Murphy AR, Oberfield SE. (2001). Fasting glucose to insulin ratio is a simple and useful measure of insulin resistance in young girls. The Journal of Clinical Endocrinology \& Metabolism 86:2863-8.

[17] Hanley AJ, Stern MP, Williams K, Haffner SM. (2002). Homeostasis model assessment of insulin resistance in relation to the incidence of cardiovascular disease. Diabetes care 25:1177-84.

[18] Jeng KC, Hou RC, Wang JC, Ping LI. (2005). Sesamin inhibits lipopolysaccharide-induced cytokine production by suppression of p38 mitogen-activated protein kinase and nuclear factor-kappaB. Immunology Letters 97:101-6.

[19] Bierhaus A, Schiekofer S, Schwaninger M, Andrassy M, Humpert PM, Chen J, Hong M, Luther T, Henle T, Klöting I, Morcos M, Hofmann M, Tritschler H, Weigle B, Kasper M, Smith M, Perry G, Schmidt AM, Stern DM, Häring HU, Schleicher E, Nawroth PP. (2001). Diabetes-associated sustained activation of the transcription factor nuclear factorkappaB. Diabetes 50:2792-808.

[20] Trattner S, Ruyter B, Østbye TK, Gjøen T, Zlabek V, KamalEldin A, Pickova J. (2008). Sesamin increases alpha-linolenic acid conversion to docosahexaenoic acid in atlantic salmon (Salmo salar L.) hepatocytes: role of altered gene expression. Lipids 43:999-1008.

[21] Du Y, Tang J, Li G, Berti-Mattera L, Lee CA, Bartkowski D, Gale D, Monahan J, Niesman MR, Alton G, Kern TS. (2005). Effects of p38 MAPK inhibition on early stages of diabetic retinopathy and sensory nerve function. Immunology Letters 97:101-6.

[22] Ohshima K, Mogi M, Horiuchi M. (2012). Role of Peroxisome Proliferator-Activated Receptor- $\gamma$ in Vascular Inflammation. International Journal of Vascular Medicine 2012:508416.

[23] Siqueira A, João FM. (2008). Effect of defatted sesame (Sesamum indicum L.) flour on the blood glucose level in type 2 diabetic women. Revista brasileira de farmacognosia 18:7783 .

[24] Sankar D, Rao MR, Sambandam G, Pugalendi KV. (2006). Effect of sesame oil on diuretics or Beta-blockers in the modulation of blood pressure, anthropometry, lipid profile, and redox status. Yale Journal of Biology and Medicine 79:1926.

[25] Ramesh B, Saravanan R, Pugalendi KV. (2005). Influence of sesame oil on blood glucose, lipid peroxidation, and antioxidant status in streptozotocin diabetic rats. Journal of Medicinal Food 8: 377-81. 